\title{
FILTERING PROBLEM FOR DISCRETE VOLTERRA EQUATIONS WITH COMBINED DISTURBANCES
}

\author{
A. Bashkov ${ }^{1)}$, G. De Nicolao ${ }^{2)}$, V. Kolmanovskii ${ }^{3)}$, and A. Matasov ${ }^{4,5)}$ \\ 1) Faculty of Applied Mathematics and Physics, Moscow Aviation Institute \\ Volokolamskoe shosse, 4, Moscow 125871 Russia, \\ 2) Dipartimento di Informatica e Sistemistica, Università di Pavia \\ via Ferrata, 1, Pavia 27100 Italy, \\ 3) Moscow Institute of Electronics and Mathematics \\ Bolshoi Vuzovskii per., 3/12, Moscow 109028 Russia, \\ 4) Faculty of Mechanics and Mathematics, Lomonosov Moscow State University \\ Leninskie Gory, Moscow GSP-2, 119992 Russia, \\ 5) Space Research Institute, Russian Academy of Sciences \\ ul. Profsoyuznaya, 84/32, Moscow GSP-7, $11799^{77}$ Russia
}

\begin{abstract}
A minimax filtering problem for discrete Volterra equations with combined noise models is considered. The combined models are defined as the sums of uncertain bounded deterministic functions and stochastic white noises. However the corresponding variational problem turns out to be very difficult for direct solution. Therefore simplified filtering algorithms are developed. The levels of nonoptimality for these simplified algorithms are introduced. In opposite to the original variational problem, these levels can be easily evaluated numerically. Thus simple filtering algorithms with guaranteed performance are obtained. Numerical experiments confirm the efficiency of our approach. Copyright (c)2005 IFAC
\end{abstract}

Keywords: discrete-time systems, filtering problem, duality

\section{INTRODUCTION}

Volterra equations are used for modelling a lot of various processes in viscoelasticity theory, demography, industrial inventory problems, physiology, polography, control of motion, aerospace technologies, etc. The various filtering problems for Volterra equations are of major interest for applications and should have a significant place in the theory of such equations. Note that the filtering problems for Volterra equations are scantily known.
In the classical filtering problem statement the plant and measurement disturbances are discrete white noises, i.e. sequences of independent random variables. However, in most applications the system contains some additional non-stochastic noises. These unknown signals can be described by set-membership models. So, a filtering problem is considered with more realistic (compared to the classical framework) assumptions, namely that the plant and measurement disturbances are the sums of white noises and uncertain bounded deterministic functions. Such disturbances are 
called combined noises. Our goal is to estimate a specified linear combination of a plant state at a given terminal instant $T$ using a linear estimator fed by observations on the segment $[0, T]$. Since the disturbances contain uncertain deterministic signals, the estimation performance is determined by the maximal value of the square root of the second moment of estimation error. The maximization is taken over all deterministic uncertainties; this maximal value is called the guaranteed estimation error. The optimal filtering problem is to find an estimator that provides a minimal value of the guaranteed estimation error. Such approach to filtering goes back to the works by N.N. Krasovskii, M.L. Lidov, I.Ya. Katz and A.B. Kurzhanskii, F.C. Schweppe, H.S. Witsenhausen, J. Sacks and D. Ylvisaker.

The guaranteed estimation error can be calculated explicitly and the optimal filtering problem can be reduced to a convex variational problem in which a convex functional is minimized on an affine manifold. However, the functional is nonsmooth, the problem dimension is high and thus the variational problem is extremely difficult.

Thus, we have to consider simplified filtering algorithms as an approximation to the unknown optimal algorithm. The level of nonoptimality is defined as the ratio of the filtering performances for the simplified and optimal estimators. Since the original variational problem cannot be solved, it is important to evaluate a level of nonoptimality without solving the original problem (Matasov, 1999). In order to obtain the desired estimate for the level of nonoptimality the duality theory of convex variational problem is employed (Ekeland and Temam, 1976). Three simplified algorithms will be studied: an algorithm of the least-squares type and two quasi-impulse algorithms. The numerical experiments will show that the levels of nonoptimality can be acceptable and in some cases they can be quite close to unity. Therefore our approach can be very useful for practical implementation. A similar estimate for the nonoptimality level was first obtained in (Matasov, 1994) for dynamic systems described by ordinary linear differential equations. In the present paper, a system with infinitely growing memory is investigated. Moreover, quasi-impulse estimators are also exploited as simplified estimators.

\section{PROBLEM STATEMENT}

Consider a linear discrete Volterra equation

$$
x(t+1)=\sum_{k=0}^{t} A(t, k) x(k)+B(t) u(t), \quad x(0)=x_{0},
$$

where $x(t) \in \mathbf{R}^{n}$ is the system state vector; $A(t, k) \in \mathbf{R}^{n \times n}, B(t) \in \mathbf{R}^{n \times r}$ are specified matrices, $u(t)=\left(u_{1}(t), \ldots, u_{r}(t)\right)^{\prime} \in \mathbf{R}^{r}$ is a perturbation vector, $x_{0}=\left(x_{01}, \ldots, x_{0 n}\right)^{\prime} \in \mathbf{R}^{n}$ is an initial state. A prime denotes the transposition sign.

Let the measurements be made for the state vector:

$$
z(k)=H^{\prime}(k) x(k)+\varrho(k), \quad k=0, \ldots, N,
$$

where $z(k) \in \mathbf{R}^{m}$ is the measurement at an instant $k, H(k) \in \mathbf{R}^{n \times m}$ is a given matrix, and $\varrho(k)=\left(\varrho_{1}(k), \ldots, \varrho_{m}(k)\right)^{\prime} \in \mathbf{R}^{m}$ is the measurement noise.

Assume that the initial state and the system perturbations have the form

$x_{0}=\stackrel{(1)}{x_{0}}+\stackrel{(2)}{x_{0}}, \quad u(t) \stackrel{(1)}{u}(t)+\stackrel{(2)}{u}(t), \varrho(k)=\stackrel{(1)}{\varrho}(k)+\stackrel{(2)}{\varrho}(k)$, where the summands satisfy the following hypotheses:

- the entries of $\stackrel{(1)}{x_{0}}$ are uncertain deterministic numbers with bounded values, and the entries of $\stackrel{(1)}{u}(t), \stackrel{(1)}{\varrho}(k)$ are uncertain deterministic bounded functions such that

$$
\left|\stackrel{(1)}{x_{0 d}}\right| \leq \sigma_{-d}, \quad\left|\stackrel{(1)}{u}_{j}(t)\right| \leq \gamma_{j}(t), \quad\left|\stackrel{(1)}{\varrho}_{l}(k)\right| \leq \sigma_{l}(k)
$$

- the vector $\stackrel{(2)}{x_{0}}$ is a zero-mean random variable and $\stackrel{(2)}{u}(t), \stackrel{(2)}{\varrho}(k)$ are standard zero-mean white noise processes with uncertain bounded covariances such that

$$
\begin{gathered}
\mathbf{E} \stackrel{(2)}{x_{0}} \stackrel{(2)}{x}_{0}^{\prime}=\operatorname{diag}\left(c_{1}^{x}, \ldots, c_{n}^{x}\right), \\
\mathbf{E} \stackrel{(2)}{u}(t) \stackrel{(2)}{u}(s)=\operatorname{diag}\left(q_{1}^{u}(t), \ldots, q_{r}^{u}(t)\right) \delta_{t s}, \\
\mathbf{E} \stackrel{(2)}{\varrho}(k) \stackrel{(2)}{\varrho}(i)=\operatorname{diag}\left(r_{1}^{\rho}(k), \ldots, r_{m}^{\rho}(k)\right) \delta_{k i},
\end{gathered}
$$

where

$$
c_{d}^{x} \leq c_{d}, \quad q_{j}^{u}(t) \leq q_{j}(t), \quad r_{l}^{\rho}(k) \leq r_{l}(k) ;
$$

- the vector $\stackrel{(2)}{x_{0}}$ and the processes $\stackrel{(2)}{u}(t), \stackrel{(2)}{\varrho}(k)$ are assumed to be mutually independent.

In these formulas $\mathbf{E}$ denotes the expectation operator, $\delta_{s t}$ is the Kronecker delta; $\sigma_{-d}, c_{d}$ are known positive numbers and $\gamma_{j}(t), q_{j}(t), \sigma_{l}(k)$, $r_{l}(k)$ are known positive functions. We call such perturbations combined disturbances.

The filtering problem is to evaluate a scalar quantity $l_{*}=a^{\prime} x(N)$, where $a \in \mathbf{R}^{n}$ is a given vector, with the help of linear functionals

$$
\hat{l}(\Phi)=\sum_{k=0}^{N} \Phi^{\prime}(k) z(k),
$$




$$
\Phi(k)=\left(\Phi_{1}(k), \ldots, \Phi_{m}(k)\right)^{\prime} \in \mathbf{R}^{m} .
$$

Since the stochastic elements $x_{0}, u, \varrho$ have uncertain statistics, let us introduce a guaranteed estimation error by the expression

$$
d(\Phi)=\max _{x_{0}, u, \varrho}\left\{\mathbf{E}\left(\hat{l}(\Phi)-l_{*}\right)^{2}\right\}^{\frac{1}{2}}
$$

where the maximum is taken over all deterministic uncertainties in the description of $x_{0}, u, \varrho$. Then the optimal guaranteed filtering problem is to find an estimator $\Phi_{\text {opt }}$ that minimizes the guaranteed estimation error:

$$
d\left(\Phi_{\mathrm{opt}}\right)=\min _{\Phi \in \mathbf{R}^{m(N+1)}} d(\Phi) .
$$

Let us introduce the quantities:

(a) the function $\xi^{\Phi}(t)$ that, for a given $\{\Phi(t)\}_{0}^{N}$, is defined by the following difference equation:

$$
\begin{gathered}
\xi^{\Phi}(t)=\sum_{k=t}^{N-1} A^{\prime}(k, t) \xi^{\Phi}(k+1)-H(t) \Phi(t), \\
\xi^{\Phi}(N)=a-H(N) \Phi(N), \quad t=N-1, \ldots, 0 ;
\end{gathered}
$$

(b) the functional $\mathcal{D}\left(\Phi_{-}, \Phi, w\right)$ :

$$
\begin{gathered}
\mathcal{D}\left(\Phi_{-}, \Phi, w\right)=I^{2}\left(\Phi_{-}, \Phi, w\right)+\Phi_{-}^{\prime} c \Phi_{-} \\
+\sum_{k=0}^{N} \Phi^{\prime}(k) r(k) \Phi(k)+\sum_{t=0}^{N-1} w^{\prime}(t+1) q(t) w(t+1),
\end{gathered}
$$

where

$$
\begin{gathered}
I\left(\Phi_{-}, \Phi, w\right)=\sum_{d=1}^{n} \sigma_{-d}\left|\Phi_{-d}\right| \\
+\sum_{l=1}^{m} \sum_{k=0}^{N} \sigma_{l}(k)\left|\Phi_{l}(k)\right|+\sum_{j=1}^{r} \sum_{t=0}^{N-1} \gamma_{j}(t)\left|w_{j}(t+1)\right|
\end{gathered}
$$

and

$$
\begin{gathered}
\Phi_{-}=\left(\Phi_{-1}, \ldots, \Phi_{-n}\right)^{\prime} \in \mathbf{R}^{n}, \\
w(t+1)=\left(w_{1}(t+1), \ldots, w_{r}(t+1)\right)^{\prime} \in \mathbf{R}^{r}, \\
c=\operatorname{diag}\left(c_{1}, \ldots, c_{n}\right), \\
r(k)=\operatorname{diag}\left(r_{1}(k), \ldots, r_{m}(k)\right), \\
q(t)=\operatorname{diag}\left(q_{1}(t), \ldots, q_{r}(t)\right) .
\end{gathered}
$$

It can be shown that

$$
d^{2}(\Phi)=\mathcal{D}\left(\Phi_{-}(\Phi), \Phi, w(\Phi)\right)
$$

where $\Phi_{-}(\Phi)$ and $w(t+1 ; \Phi)$ are defined by the constraints

$$
\begin{aligned}
\Phi_{-}-\xi^{\Phi}(0) & =0, \\
w(t+1)-B^{\prime}(t) \xi^{\Phi}(t+1) & =0, \quad t=0, \ldots, N-1 .
\end{aligned}
$$

Thus, the optimal guaranteed filtering problem reduces to the following nonsmooth variational problem:

$$
\mathcal{D}_{0}=\min _{\Phi_{-} \Phi, w} \mathcal{D}\left(\Phi_{-}, \Phi, w\right)
$$

under constraints (2).

Unfortunately, this is a very difficult nonsmooth problem. Therefore the following approach is exploited (Matasov, 1999). Some simplified estimator $\varphi$ is searched instead of $\Phi_{\text {opt }}$ and the approximation quality is defined by the ratio

$$
\Delta=d(\varphi) / d\left(\Phi_{\mathrm{opt}}\right)
$$

Clearly $\Delta \geq 1$. However the optimal estimator $\Phi_{\text {opt }}$ is unknown and thus $\Delta$ is unknown. Therefore an upper bound $\Delta^{0}$ will be constructed for $\Delta$ that can be calculated without exactly solving the problem (3), (2). If this upper bound is not large, then $\varphi$ is acceptable for the solution of the filtering problem.

\section{LINEAR-QUADRATIC PROBLEM}

Consider an approximating linear-quadratic variational problem that will be a main tool for our analysis of the initial variational problem (3), (2). Namely, let us replace the nonsmooth functional (1) by the following quadratic functional

$$
\mathcal{J}\left(\Phi_{-}, \Phi, w\right)=\Phi_{-}^{\prime} P_{0} \Phi_{-}
$$

$$
+\sum_{k=0}^{N} \Phi^{\prime}(k) R(k) \Phi(k)+\sum_{t=0}^{N-1} w^{\prime}(t+1) Q(t) w(t+1) .
$$

Here

$$
\begin{gathered}
P_{0}=\beta_{0} \operatorname{diag}\left(\sigma_{-1}^{2}, \ldots, \sigma_{-n}^{2}\right)+c, \\
R(k)=\beta_{1} \operatorname{diag}\left(\sigma_{1}^{2}(k), \ldots, \sigma_{m}^{2}(k)\right)+r(k), \\
Q(t)=\beta_{2} \operatorname{diag}\left(\gamma_{1}^{2}(t), \ldots, \gamma_{r}^{2}(t)\right)+q(t),
\end{gathered}
$$

where $\beta_{0}, \beta_{1}$, and $\beta_{2}$ are scalar scale multipliers.

Thus the following approximating linearquadratic problem is obtained:

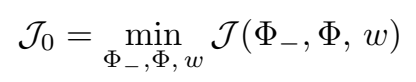

under constraints (2).

TheOrem 1. The solution $\left\{\Phi_{-}^{0}, \Phi^{0}, w^{0}\right\}$ of the linear-quadratic problem (4), (2) is given by the relations

$$
\Phi_{-}^{0}=\xi(0)
$$

$\Phi^{0}(k)=R^{-1}(k) H^{\prime}(k)\left(P(k) \xi(k)+\sum_{s=k}^{N-1} P_{1}^{\prime}(k, s) \xi(s+1)\right)$, 


$$
w^{0}(t+1)=B^{\prime}(t) \xi(t+1),
$$

where $\xi(t)$ satisfies the backward equation

$$
\begin{aligned}
\xi(t)=\sum_{k=t}^{N-1} C^{\prime}(k, t) \xi(k+1), \quad t=N-1, \ldots, 0, \\
C(t, l)=A(t, l)-D(t, l) H^{\prime}(l), \\
D(t, l)=\left[P_{1}^{\prime}(l, t)+A(t, l) P(l)\right] H(l) \\
\times\left[H^{\prime}(l) P(l) H(l)+R(l)\right]^{-1}
\end{aligned}
$$

with the terminal condition

$$
\xi(N)=\left(E+H(N) R^{-1}(N) H^{\prime}(N) P(N)\right)^{-1} a .
$$

The matrices $P(t) \geq 0$ and $P_{1}(t, k)$ that enter in (5) are determined by the system of equations

$$
\begin{array}{r}
P(t+1)=\sum_{l=0}^{t}\left[C(t, l) P_{1}(l, t)+P_{1}^{\prime}(l, t) A^{\prime}(t, l)\right. \\
\left.+C(t, l) P(l) A^{\prime}(t, l)\right]+B(t) Q(t) B^{\prime}(t), \\
P_{1}(t+1, k)=\sum_{l=0}^{t}\left[C(t, l) P_{1}(l, k)+P_{1}^{\prime}(l, t) A^{\prime}(k, l)\right. \\
\left.+C(t, l) P(l) A^{\prime}(k, l)\right]
\end{array}
$$

with the initial conditions

$P(0)=P_{0}, \quad P_{1}(0, k)=0, \quad k=0, \ldots, N-1$.

Moreover,

$$
\mathcal{J}_{0}=a^{\prime} P(N)\left(E+H(N) R^{-1}(N) H^{\prime}(N) P(N)\right)^{-1} a .
$$

So, the linear-quadratic problem (4), (2) has an exact solution that can be easily implemented. The proof of Theorem 1 is based on (Kuchkina, and Shaikhet, 1998), (Bashkov, et al., 2004), and some new argument. Note that a corresponding optimal estimate $\hat{l}\left(\Phi^{0}\right)$ has a Kalman type representation.

\section{LEVELS OF NONOPTIMALITY}

To formulate the main result let us introduce the following auxiliary quantities (that are calculated from Theorem 1):

$$
\begin{array}{cc}
s_{d}=\frac{\sigma_{-d}^{2}}{c_{d}}, & S_{d}=\frac{P_{0 d} \Phi_{-d}^{0}}{\sigma_{-d}}, \\
f_{l}(k)=\frac{\sigma_{l}^{2}(k)}{r_{l}(k)}, & F_{l}(k)=\frac{R_{l}(k) \Phi_{l}^{0}(k)}{\sigma_{l}(k)},
\end{array}
$$

$g_{j}(t)=\frac{\gamma_{j}^{2}(t)}{q_{j}(t)}, \quad G_{j}(t)=\frac{Q_{j}(t) w_{j}^{0}(t+1)}{\gamma_{j}(t)}$,

where

$$
\begin{gathered}
P_{0}=\operatorname{diag}\left(P_{01}, \ldots, P_{0 n}\right), \\
R(k)=\operatorname{diag}\left(R_{1}(k), \ldots, R_{m}(k)\right), \\
Q(t)=\operatorname{diag}\left(Q_{1}(t), \ldots, Q_{r}(t)\right) .
\end{gathered}
$$

Let $\varphi$ be any simplified estimator. The main result is given by the following theorem.

THEOREM 2. The level of nonoptimality for the simplified estimator $\varphi$ is evaluated by the following inequalities:

$1 \leq \Delta \leq \Delta^{0}=\frac{\mathcal{D}^{\frac{1}{2}}\left(\varphi_{-}, \varphi, w^{\varphi}\right) \cdot \Omega^{\frac{1}{2}}\left(\Phi_{-}^{0}, \Phi^{0}, w^{0}\right)}{\mathcal{J}_{0}}$,

where $\mathcal{D}\left(\varphi_{-}, \varphi, w^{\varphi}\right)$ is given by $(1), \varphi_{-}=\xi^{\varphi}(0)$ and $w^{\varphi}(t+1)=B^{\prime}(t) \xi^{\varphi}(t+1)$ are set by constraints $(2), \mathcal{J}_{0}$ is specified by (6), and

$$
\begin{aligned}
& \Omega\left(\Phi_{-}^{0}, \Phi^{0}, w^{0}\right)=\sum_{\substack{d:\left|S_{d}\right|>\zeta^{0} \\
d=1, \ldots, n}} s_{d}\left(\left|S_{d}\right|-\zeta^{0}\right)^{2} \\
& +\sum_{l=1}^{m} \sum_{\substack{k:\left|F_{l}(k)\right|>\zeta^{0} \\
k=0, \ldots, N}} f_{l}(k)\left(\left|F_{l}(k)\right|-\zeta^{0}\right)^{2} \\
& +\sum_{j=1}^{r} \sum_{\substack{t:\left|G_{j}(t)\right|>\zeta^{0} \\
t=0, \ldots, N-1}} g_{j}(t)\left(\left|G_{j}(t)\right|-\zeta^{0}\right)^{2}+\zeta^{0^{2}}
\end{aligned}
$$

The value $\zeta^{0}$ satisfies the equation $\mathcal{E}(\zeta)=0$ on the segment $\left[0, \zeta_{\max }\right]$, where

$$
\begin{aligned}
& \mathcal{E}(\zeta)=\sum_{\substack{d:\left|S_{d}\right|>\zeta \\
d=1, \ldots, n}} s_{d}\left(\left|S_{d}\right|-\zeta\right) \\
& +\sum_{l=1}^{m} \sum_{\substack{k:\left|F_{l}(k)\right|>\zeta \\
k=0, \ldots, N}} f_{l}(k)\left(\left|F_{l}(k)\right|-\zeta\right) \\
& +\sum_{j=1}^{r} \sum_{\substack{t:\left|G_{j}(t)\right|>\zeta \\
t=0, \ldots, N-1}} g_{j}(t)\left(\left|G_{j}(t)\right|-\zeta\right)-\zeta
\end{aligned}
$$

and $\zeta_{\max }$ is the maximum out of three quantities:

$$
\max _{d=1, \ldots, n}\left|S_{d}\right|, \max _{\substack{l=1, \ldots, m \\ k=0, \ldots, N}}\left|F_{l}(k)\right|, \max _{\substack{j=1, \ldots, r \\ t=0, \ldots, N-1}}\left|G_{j}(t)\right| .
$$

The proof is essentially based on the duality theory for convex variational problems.

Similarly to (Matasov, 1999) one can prove that the function $\mathcal{E}(\zeta)$ is continuous, strictly 
monotonically decreasing and has different signs at the endpoints of $\left[0, \zeta_{\max }\right]$. Moreover, it is almost obvious that $\mathcal{E}(\zeta)$ is a piecewise linear and convex function. Therefore the equation $\mathcal{E}(\zeta)=0$ has a unique root, which can easily be found by sequentional halving the interval.

It follows from Theorem 2 that $\Delta^{0}$ can be easily calculated without exactly solving the original complex minimax filtering problem.

Let us consider three simplified estimators $\varphi$ and the corresponding values of $\Delta^{0}$ calculated from Theorem 2.

\subsection{Least-squares estimator}

First consider the estimator $\Phi^{0}(k)$ obtained from the solution of the approximating linear-quadratic problem (4), (2). We call it the least-squares estimator and denote the corresponding value of $\Delta^{0}$ by $\Delta_{L S}^{0}$.

\subsection{Quasi-impulse estimators}

Consider the original variational problem (3), (2). The Lagrange function for (3), (2) has the form

$$
\begin{gathered}
\mathcal{L}_{\mathcal{D}}\left(\Phi_{-}, \Phi, w ; \lambda, p\right)=\mathcal{D}\left(\Phi_{-}, \Phi, w\right)+\lambda^{\prime}\left\{\xi^{\Phi}(0)-\Phi_{-}\right\} \\
+\sum_{t=0}^{N-1} p^{\prime}(t+1)\left\{B^{\prime}(t) \xi^{\Phi}(t+1)-w(t+1)\right\} .
\end{gathered}
$$

In accordance with the duality theory (Ekeland and Temam, 1976) the dual problem for (3), (2) is the maximization problem

$$
\max _{\lambda, p}\left(\min _{\Phi-, \Phi, w} \mathcal{L}_{\mathcal{D}}\left(\Phi_{-}, \Phi, w ; \lambda, p\right)\right) .
$$

Moreover, if $\left(\lambda^{\mathrm{opt}}, p^{\mathrm{opt}}\right)$ is the solution for (10), then the solution of the original problem $\left(\Phi_{- \text {opt }}, \Phi_{\text {opt }}, w_{\text {opt }}\right)$ yields the minimum in $\left(\Phi_{-}, \Phi, w\right)$ for the Lagrange function $\mathcal{L}_{\mathcal{D}}\left(\Phi_{-}, \Phi, w ; \lambda^{\mathrm{opt}}, p^{\mathrm{opt}}\right)$. However, the dual problem (10) is as complex as the original one, and $\left(\lambda^{\text {opt }}, p^{\text {opt }}\right)$ are unknown.

Let us consider the Lagrange function $\mathcal{L}_{\mathcal{J}}$ for the approximating linear-quadratic problem (4), (2) that is similar to $\mathcal{L}_{\mathcal{D}}$ but with $\mathcal{D}$ replaced by $\mathcal{J}$. The corresponding dual problem is defined by (10) but with $\mathcal{L}_{\mathcal{D}}$ replaced by $\mathcal{L}_{\mathcal{J}}$. In opposite to $(10)$ the dual problem to the linear-quadratic problem has an explicit solution

$$
\lambda^{0}=2 P_{0} \xi(0), \quad p^{0}(t+1)=2 Q(t) \xi(t+1),
$$

where $\xi(t)$ is defined by (5). The multipliers (11) prompt us a successful direction for the approximation $\left(\lambda^{\mathrm{a}}, p^{\mathrm{a}}\right)$ to the unknown $\left(\lambda^{\mathrm{opt}}, p^{\mathrm{opt}}\right)$ in the form $\left(\lambda^{\mathrm{a}}, p^{\mathrm{a}}\right)=\nu\left(\lambda^{0}, p^{0}\right)$, where $\nu$ is a positive scalar. Then the dual problem (10) can be approximated by the problem

$$
\max _{\nu>0}\left(\min _{\Phi-_{-}, \Phi, w} \mathcal{L}_{\mathcal{D}}\left(\Phi_{-}, \Phi, w ; \nu \lambda^{0}, \nu p^{0}\right)\right) .
$$

One can show after laborious calculation that the last problem has an explicit solution

$$
\nu^{0}=\mathcal{J}_{0} \Omega^{-1}\left(\Phi_{-}^{0}, \Phi^{0}, w^{0}\right)
$$

where $\mathcal{J}_{0}$ and $\Omega$ are defined by (6) and (7). So the multipliers $\left(\nu^{0} \lambda^{0}, \nu^{0} p^{0}\right)$ can be considered as a desired approximation for the unknown $\left(\lambda^{\mathrm{opt}}, p^{\mathrm{opt}}\right)$. Thus in accordance with the duality theory a simplified estimator can be obtained from the minimization of $\mathcal{L}_{\mathcal{D}}\left(\Phi_{-}, \Phi, w ; \nu^{0} \lambda^{0}, \nu^{0} p^{0}\right)$ in $\left(\Phi_{-}, \Phi, w\right)$. This simplified estimator has the form $\hat{\Phi}(k)=\left(\hat{\Phi}_{1}(k), \ldots, \hat{\Phi}_{m}(k)\right)^{\prime}$, where $\hat{\Phi}_{l}(k)$ is determined by the expression

$\left\{\begin{array}{lrl}\nu^{0} r_{l}^{-1}(k)\left(R_{l}(k) \Phi_{l}^{0}(k)-\zeta^{0} \sigma_{l}(k)\right), & F_{l}(k) & >\zeta^{0} \\ 0, & \left|F_{l}(k)\right| \leq \zeta^{0} \\ \nu^{0} r_{l}^{-1}(k)\left(R_{l}(k) \Phi_{l}^{0}(k)+\zeta^{0} \sigma_{l}(k)\right), & F_{l}(k)<-\zeta^{0} .\end{array}\right.$

Here $\nu^{0}$ is set by $(12), \Phi^{0}(k)$ is given by Theorem 1 , and $\zeta^{0}$ is defined by $(8)$.

Obviously the estimator $\hat{\Phi}_{l}(k)$ has a "dead" zone, that is $\hat{\Phi}(k)$ is of quasi-impulse type. So we call $\hat{\Phi}(k)$ the quasi-impulse estimator and denote the corresponding nonoptimality level by $\Delta_{Q I}^{0}$. Note that this estimator has the same structure as the unknown optimal one. Thus we could hope that the quasi-impulse estimator would be useful.

Unfortunately, the values of $\Delta_{Q I}^{0}$ turned out to be too large in many cases, and the quasi-impulse estimator $\hat{\Phi}(k)$ frequently is not acceptable. Therefore we have to modify it basing on the feature that $\hat{\Phi}_{l}(k)=0$ for

$$
\left|F_{l}(k)\right| \leq \zeta^{0} .
$$

This feature means that the measurements $z_{l}(k)$ are ignored for instants $k$ such that (13) holds. By analogy, let us introduce a parametrized estimator $\Phi^{\zeta}(k)$ that is provided by a special least-squares estimator $\Phi^{0}(k)$ based on a reduced set of measurements in which we ignore the measurements $z_{l}(k)$ such that $\left|F_{l}(k)\right| \leq \zeta$, where $\zeta$ is a given parameter. We call this estimator the modified quasi-impulse estimator and denote the corresponding nonoptimality level by $\Delta_{M Q I}^{0}$. Obviously, for $\zeta=0$ the estimator $\Phi^{\zeta}$ coincides with the least-squares estimator $\Phi^{0}$.

Note that our simplified estimators implicitly depend on three scalar parameters $\beta_{0}, \beta_{1}, \beta_{2}$ (the 
Table 1. Levels for $\sigma=1, \quad r=25 \quad(w=1)$

\begin{tabular}{cccccc}
$q^{\frac{1}{2}}=5 \gamma$ & $2 \cdot 10^{-4}$ & $2 \cdot 10^{-3}$ & $2 \cdot 10^{-2}$ & $2 \cdot 10^{-1}$ & $2 \cdot 10^{0}$ \\
\hline$\Delta_{L S}^{0}$ & 1.19 & 1.23 & 1.30 & 1.25 & 1.09 \\
$\Delta_{M Q I}^{0}$ & 1.07 & 1.11 & 1.28 & 1.20 & 1.08 \\
\hline
\end{tabular}

Table 2. Levels for $\sigma=1, \quad r=1 \quad(w=1)$

\begin{tabular}{cccccc}
$q^{\frac{1}{2}}=\gamma$ & $4 \cdot 10^{-5}$ & $4 \cdot 10^{-4}$ & $4 \cdot 10^{-3}$ & $4 \cdot 10^{-2}$ & $4 \cdot 10^{-1}$ \\
\hline$\Delta_{L S}^{0}$ & 1.98 & 2.06 & 2.04 & 2.23 & 1.98 \\
$\Delta_{M Q I}^{0}$ & 1.24 & 1.36 & 1.80 & 2.07 & 1.92 \\
\hline
\end{tabular}

modified quasi-impulse estimator depends on parameter $\zeta$ as well) that can be adjusted to improve the level of nonoptimality. An obvious starting choice is $\beta_{0}=\beta_{1}=\beta_{2}=1\left(\zeta=\zeta^{0}\right)$. It should be emphasized that all estimators are easy for calculation. Thus we constructed parametric families of suboptimal algorithms with a moderate number of parameters that can be easily "tuned in the wave" of a specific filtering problem with a guaranteed evaluation of filtering performance.

\section{NUMERICAL EXPERIMENTS}

Consider a two-dimensional Volterra equation

$$
\begin{gathered}
x(t+1)=\sum_{k=0}^{t} \alpha^{t-k+1}\left(\begin{array}{ll}
w & 1 \\
0 & 1
\end{array}\right) x(k)+u(t), \\
x(k)=\left(x_{1}(k), x_{2}(k)\right)^{\prime}, \quad u(t)=\left(u_{1}(t), u_{2}(t)\right)^{\prime}
\end{gathered}
$$

and scalar measurements

$$
z(k)=x_{1}(k)+\varrho(k), \quad k=0, \ldots, N .
$$

The aim is to estimate the second component $x_{2}(N)$. Here $\alpha$ and $w$ are specified scalars. The uncertainties in initial state and disturbances are described in Section 2. We put $N=100, \alpha=0.5$, $w=1$ or $w=0.8, \sigma_{-1}=\sigma_{-2}=10, c_{1}=c_{2}=100$, $\gamma_{1}(t)=\gamma_{2}(t)=\gamma, q_{1}(t)=q_{2}(t)=q, \sigma_{1}(k)=$ $\sigma_{2}(k)=\sigma, r_{1}(k)=r_{2}(k)=r$. The optimized (in $\beta_{1}, \beta_{2}$ and $\left.\zeta ; \beta_{0}=1\right)$ values of $\Delta_{L S}^{0}$ and $\Delta_{M Q I}^{0}$ for various noise intensities are shown in Table 1 , Table 2, and Table 3. Parameter $\beta_{0}$ is not varied since it has no virtual effect on $\Delta^{0}$.

As a rule, the optimized values of $\Delta_{L S}^{0}$ differ only weakly from their not optimized values

Table 3. Levels for $\sigma=1, \quad r=1 \quad(w=0.8)$

\begin{tabular}{cccccc}
$q^{\frac{1}{2}}=\gamma$ & $4 \cdot 10^{-5}$ & $4 \cdot 10^{-4}$ & $4 \cdot 10^{-3}$ & $4 \cdot 10^{-2}$ & $4 \cdot 10^{-1}$ \\
\hline$\Delta_{L S}^{0}$ & 1.09 & 1.13 & 1.42 & 2.09 & 1.90 \\
$\Delta_{M Q I}^{0}$ & 1.03 & 1.05 & 1.09 & 1.41 & 1.87 \\
\hline
\end{tabular}

(i.e. with $\beta_{0}=\beta_{1}=\beta_{2}=1$ ) which are also acceptable. At the same time, the values of $\Delta_{M Q I}^{0}$ usually are very sensitive to optimization. Note that the contribution of uncertain deterministic disturbances into estimation error far exceeds the contribution of white noise components of the same intensities $\left(\sigma=r^{\frac{1}{2}}, \gamma=q^{\frac{1}{2}}\right)$. So, in the case presented in Table 1 the influence of deterministic components is essential and cannot be neglected in advance. It follows from the tables that the least-squares estimator is quite acceptable and, in some cases, it is near-optimal. Moreover, sometimes the modified quasi-impulse estimator can improve markedly the filtering performance. Thus the simplified estimators can be successfully used for filtering in Volterra equations.

\section{CONCLUSION}

In the paper, the optimal minimax filtering problem with combined disturbances for linear discrete Volterra equations was considered. The corresponding variational problem turned out to be very difficult. Then the least-squares and the quasi-impulse simplified estimators were proposed as approximate solutions. The levels of nonoptimality for these simplified estimators were derived. They can be easily calculated without exactly solving the original filtering problem. Thus a useful constructive approach has been developed for the minimax filtering problem for discrete Volterra equations.

The work was supported by a grant of the Cariplo Foundation (Italy).

\section{REFERENCES}

Bashkov A.B., Kolmanovskii V.B., Mao X., Matasov A.I. (2004). Mean-square filtering problem for discrete Volterra equations. Journal of Stochastic Analysis and Applications, 22, pp. 1085-1110.

Ekeland, I. and R. Temam (1976). Convex Analysis and Variational Problems. North-Holland, Amsterdam; Elsevier, New York.

Kuchkina N., Shaikhet L. (1998). Optimal estimation of stochastic difference equations. Proceedings of the CESA'98. Symposium on Signal Processing and Cybernetics, Tunisia, April 1-4, 4, pp. 165-169.

Matasov A.I. (1994). The Kalman-Bucy filter accuracy in the guaranteed parameter estimation problem with unknown statistics. IEEE Transactions on Automatic Control, AC-39, pp. 635-639.

Matasov, A.I. (1999). Estimators for Uncertain Dynamic Systems, Kluwer Academic Publishers, Dordrecht-Boston-London. 